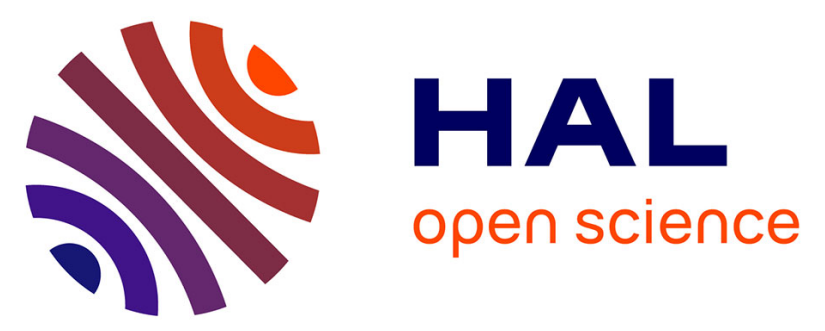

\title{
Computational Fluid Dynamics and Experimental Validations of the Direct Coupling Between Interior, Intermediate and Exterior Ballistics Using the Euler Equations
}

Roxan Cayzac, Eric Carette, Thierry Alziary de Roquefort, François-Xavier Renard, Dominique Roux, Jean-Noël Patry, Patrick Balbo

\section{To cite this version:}

Roxan Cayzac, Eric Carette, Thierry Alziary de Roquefort, François-Xavier Renard, Dominique Roux, et al.. Computational Fluid Dynamics and Experimental Validations of the Direct Coupling Between Interior, Intermediate and Exterior Ballistics Using the Euler Equations. Journal of Applied Mechanics, 2011, Volume 78, pp.061006-1/061006-17. hal-00627597

\section{HAL Id: hal-00627597 \\ https://hal.science/hal-00627597}

Submitted on 29 Sep 2011

HAL is a multi-disciplinary open access archive for the deposit and dissemination of scientific research documents, whether they are published or not. The documents may come from teaching and research institutions in France or abroad, or from public or private research centers.
L'archive ouverte pluridisciplinaire HAL, est destinée au dépôt et à la diffusion de documents scientifiques de niveau recherche, publiés ou non, émanant des établissements d'enseignement et de recherche français ou étrangers, des laboratoires publics ou privés. 


\title{
Computational Fluid Dynamics and Experimental Validations of the Direct Coupling Between Interior, Intermediate and Exterior Ballistics Using the Euler Equations
}

\author{
Dr. R. Cayzac ${ }^{1}$, E. Carette ${ }^{1}$, Pr. T. Alziary de Roquefort ${ }^{2}$, F. X. Renard $^{3}$, D. Roux ${ }^{3}$, J. N. Patry ${ }^{3}$ and P. Balbo $^{3}$ \\ ${ }^{1}$ Nexter Munitions, Technical Direction, 7 Route de Guerry, 18023 Bourges Cedex, France, T: +332 48219523 , F: + 33248219406 , \\ E: r.cayzac@nexter-group.fr \\ ${ }^{2}$ Expert consulting in Fluid Mechanics (retired Professor of the University of Poitiers), 129 Rue des Quatres Roues, 86000, Poitiers, \\ France, T: +33549889662, E: thierry.alziary@ club-internet.fr \\ ${ }^{3}$ Nexter Systems, Weapons Systems Department, 7 Route de Guerry, 18023 Bourges Cedex, France, T: +332 48219023 , F: + 33248 \\ 2199 05, E: d.roux@ nexter-group.fr
}

\begin{abstract}
For several years we have been working on the development of a CFD ballistics code called FREIN. This code is the result of a strong cooperation between Nexter Munitions and the University of Poitiers. In the last years, efforts have been carried out to improve the 3D modeling. In a fully unsteady way, the interior, intermediate and exterior ballistics were modeled as well as the weapon system environment. The complex phenomena encountered are investigated by an adapted numerical simulation approach using the Euler equations for two immiscible gases. The method involves moving bodies with respect to fixed Cartesian meshes and the aerodynamic forces are used to compute the trajectories. In this paper, theoretical developments and computations have been applied mainly to the simulation of the firing of an advanced $120 \mathrm{~mm}$ lightweight tank demonstrator. In comparison with firing experiments, first computation validation results concerning interior ballistics, muzzle brake flow, sabot discard and blast wave propagation and reflection are presented and are very satisfactory.
\end{abstract}

Keywords: interior ballistics, intermediate ballistics, exterior ballistics, Euler equations, computational fluid dynamics, muzzle brake, sabot separation, blast wave

\section{Introduction}

Progress in CFD capabilities make the numerical simulation of ballistic phenomena very tempting. However this is a three-dimensional unsteady aerodynamic problem with several mutually interacting moving bodies and very different flow regimes. In view of the high cost of such computations one must accept some simplifications. So far it seems that most attempts have considered steady situations with Chimera mesh based solvers [4, 7]. The purpose of the present paper is to investigate the coupling of the intermediate ballistics problem with the interior and exterior ballistic flows. To treat the muzzle brake flow we have chosen to investigate the use of Cartesian grids because a body fitted mesh generation around an object like a muzzle brake would be difficult and too time consuming. The modeling and the numerical methods used for the internal and external aerodynamic problems are introduced in section II. The coupling between the two problems and with the dynamic trajectory calculation is introduced in section III. Several validation results are given in section IV.

\section{Modeling and Numerical Methods}

As explained such computations are so expensive that, in a first step, we must make some simplifications: the computations presented in this paper are based only on the Euler equations. 


\subsection{Internal Ballistics}

The internal ballistics between the breech and the projectile is a heterogeneous two phase reacting flow problem which involve a number of physical modelings for the inter-phase drag and heat transfer, the form function of the particles, the rate of surface regression and the granular stress. For all these terms we use models close to those of reference [9] in the framework of a 1D, two-phase approximation [6], in a tube of variable cross section $\mathrm{A}(\mathrm{x}, \mathrm{t})$. The cross section is a function of time because one must take into account the tail of the moving projectile between the breech and the obturator. Let $\phi$ be the volume fraction of the gas of density, $\rho$, velocity, $u$, total energy, E, and $\widetilde{\rho}$ the density of the continuous phase representing the propellant grains of velocity $\tilde{u}$.

The balance equations are:

$$
\begin{aligned}
& \frac{\partial[\rho \cdot \phi \cdot A]}{\partial t}+\frac{\partial[\rho \cdot u \cdot \phi \cdot A]}{\partial x}=G \cdot A+G_{i} \\
& \frac{\partial[\rho \cdot u \cdot \phi \cdot A]}{\partial t}+\frac{\partial\left[\rho \cdot u^{2} \cdot \phi \cdot A+p \cdot \phi \cdot A\right]}{\partial x}=[G \cdot \tilde{u}-f] \cdot A+p \cdot \frac{\partial[\phi \cdot A]}{\partial x} \\
& \frac{\partial[\rho \cdot E \cdot \phi \cdot A]}{\partial t}+\frac{\partial[\rho \cdot u \cdot H \cdot \phi \cdot A+p \cdot \phi \cdot A]}{\partial x}=-p \cdot \frac{\partial[\phi \cdot A]}{\partial x}+\left[G \cdot\left(E_{c}+\frac{\tilde{u}^{2}}{2}+\frac{p}{\rho}\right)-\tilde{u} \cdot f \cdot A_{s} \cdot q_{t}\right] \cdot A+G_{i} \cdot E_{c}+\frac{\partial[\tilde{\rho} \cdot(1-\phi) \cdot A]}{\partial t}+\frac{\partial[\tilde{\rho} \cdot \tilde{u} \cdot(1-\phi) \cdot A]}{\partial x}=-G \cdot A \\
& \frac{\partial[\tilde{\rho} \tilde{u} \cdot(1-\phi) \cdot A]}{\partial t}+\frac{\partial\left[\tilde{\rho} \cdot \tilde{u}^{2} \cdot(1-\phi) \cdot A+(1-\phi)\left(p+p^{*}\right) \cdot A\right]}{\partial x}=[-G \cdot \tilde{u}+f]+\left(p+p^{*}\right)+\frac{\partial[(1-\phi) \cdot A]}{\partial x} .
\end{aligned}
$$

Where $\mathrm{G}$ is the mass flux given by the combustion of the propellant grains, $f$ the inter-phase drag, $A_{s}$ the specific area (area per unit volume) of the propellant grains, $q_{t}$ the inter-phase heat flux, $E_{c}$ the energy furnished by the combustion and $G_{i}$ the mass flow rate of the igniter. These equations are supplemented by two equations for a variable linked to the temperature of the propellant grains and the burned distance, both quantities being convected with the propellant phase. The gas follows a Noble-Abel equation of state:

$$
p=\frac{\gamma-1}{1-b . \rho} . \rho . e
$$

Where $\mathrm{b}$ is the covolume and the internal energy e is a function of the temperature $\mathrm{e}=\mathrm{Cv}$.T.

It is well known [9], [17], [14] that the system of equations (1) is not totally hyperbolic and that the initial value problem is therefore mathematically ill-posed. Although the system can be regularized by introduction of a pressure correction, as done for instance by Sainsaulieu [16], we have followed the current practice in internal ballistics which is to admit that the behavior of the source terms leads to a well-posed initial value problem. Taking into account the strong similarity between some terms containing the volume fraction $\phi$ which is part of the solution and the cross section A which can be considered as a prescribed quantity, we have treated the terms $p \cdot \frac{\partial[\phi . A]}{\partial x}$ in the gas momentum equation and $-p \cdot \frac{\partial[\phi . A]}{\partial t}$ in the gas energy equations as source terms. The left hand side of system (1) is then an hyperbolic system in conservative form. Its eigenvalues can be computed and a Roe type approximate Riemann solver can be built and used in a classical flux difference splitting approach.

We used a fixed grid approach where the $\mathrm{x}$ boundaries of the cells are fixed except for the last one adjacent to the obturator of the projectile. However the volume of the cells may vary with the time either because of the displacement of the tail of the projectile for the standard cells or because of the displacement of the airtight section for the last cell. A new cell is added when the $\mathrm{x}$ size of the cell adjacent to the projectile is larger than 1.5 times the size of a standard cell. All the volumes are computed exactly in order to insure a strict conservation of mass, momentum, and energy which is an essential feature for internal ballistics. At the beginning of the simulation we use typically 80 cells between the breech and the projectile. When the projectile moves in the tube, the number of active cells increases and remeshing by merging every two cells is performed above some given threshold for the number of cells provided that the cell size $\Delta \mathrm{x}$ is not larger than a given value. For typical guns the number of active cells when the projectile leaves the tube is about 800 . 
With the same kind of unsteady one-dimensional approach we also compute the precursor flow in the tube between the projectile and the gun muzzle. This gives a direct evaluation of the counter pressure acting on the projectile. The friction of the projectile in the tube is usually modeled by empirical laws which take into account this counter pressure and must therefore be modified because the counter pressure is now directly computed and taken into account in the evaluation of the force acting on the projectile.

\subsection{Intermediate Ballistics}

The external ballistics problem involves both the propellant gas and the atmosphere. We assume that these two gas are inviscid, immiscible and therefore separated by an interface. The location of this contact discontinuity is treated by the level set method introduced by Mulder, Osher and Sethian [12], that is the interface correspond to the level set $\psi=0$ of a hypersurface $\psi(\mathrm{x}, \mathrm{y}, \mathrm{z}, \mathrm{t})$ which must satisfy the following evolution equation in conservation form :

$$
\frac{\partial \psi}{\partial t}+\frac{\partial \rho \cdot u \cdot \psi}{\partial x}+\frac{\partial \rho \cdot v \cdot \psi}{\partial y}+\frac{\partial \rho \cdot w \cdot \psi}{\partial z}=0
$$

We take $\psi<0$ in the propellant gas and $\psi>0$ in the atmosphere. Both gas satisfy the Euler equations. The equations of state are $\mathrm{p}=\left(\gamma_{\mathrm{a}}-1\right)$. $\rho$.e in the atmosphere and the Noble-Abel equation $\mathrm{p}=\Gamma(\rho)$. $\rho$.e with $\Gamma=\left(\gamma_{p}-1\right) /(1-b . \rho)$ in the propellant gas. This multifluids problem is solved by an algorithm introduced by Abgrall and Karni [1]. The conserved variables $Q=(\rho, \rho u, \rho v, \rho w, \rho E, \rho \psi)$ are computed in each cell using the appropriate equation of state given by $\psi$. If there is an interface between two cells, two intercell fluxes must be computed, one using $\gamma_{\mathrm{a}}$ which is used for the air cell and one using $\gamma_{\mathrm{p}}$ which is used for the propellant gas cell. After updating the variables at time $\mathrm{n}+1$ one compute the pressure according to the frozen value $\psi^{\mathrm{n}}$. Then the new value $\psi^{\mathrm{n}+1}$ of the level set function is computed. This algorithm ensures a strict conservation of mass and momentum and introduces only a small error in the total energy balance.

We use this algorithm in the framework of a second order MUSCL approach with Van Albada limiter on primitive variables and a ROE solver. The Roe method [15] is based on the following averages :

$$
\begin{aligned}
& \bar{f}=\alpha \cdot f^{-}+(1-\alpha) \cdot f^{+} \\
& f=(1-\alpha) \cdot f^{-}+\alpha \cdot f \\
& \text { with } \quad \alpha=\frac{\sqrt{\rho^{-}}}{\sqrt{\rho^{-}}+\sqrt{\rho^{+}}}
\end{aligned}
$$

which have the properties :

$$
\begin{aligned}
& \Delta(f \cdot g)=(f) \cdot \Delta g+\bar{g} \cdot \Delta f \\
& \rho . f=\rho \cdot \bar{f}
\end{aligned}
$$

Using these properties we can show that for a Noble- Abel equation the term $\Gamma(\rho)$ must be averaged as $\underline{\Gamma}$ and that in the speed of sound given by

$$
c^{2}=\Gamma \cdot\left[H-\frac{u^{2}+v^{2}+w^{2}}{2}+\frac{p \cdot b}{\gamma-1}\right]
$$


the term p.b/( $\gamma-1)$ must be averaged as :

$$
\frac{p \cdot b}{\gamma-1}=\frac{p}{\Gamma^{2}} \cdot \frac{\partial \Gamma}{\partial \rho}=\frac{1}{\Gamma} \cdot \frac{p}{\Gamma} \cdot \frac{\Delta \Gamma}{\Delta \rho}
$$

If $\Delta \rho$ is too small the ratio $\Delta \Gamma / \Delta \rho$ is replaced by the analytic derivative of $\Gamma(\rho)$ evaluated at the Roe average $\rho$. The time integration is performed with a second order two step predictor-corrector method. As shown on Fig. 1, the pressure at the gun muzzle, when the projectile leaves the tube, is about 1000 bars and the numerical scheme must be able to compute very strong shocks and expansions. However a classical ROE flux difference splitting solver usually fails on very strong expansions, for instance when one state is near vacuum like in test case 2 of reference [11]. As robustness is essential we used a modified version of the Roe scheme, introduced by Kim et al. [10], which avoids the use of an entropy fix parameter. With this scheme we were able to run all the test cases of reference [11] without problem and with good quality solutions.

\subsection{Cartesian Grids and Boundary Conditions}

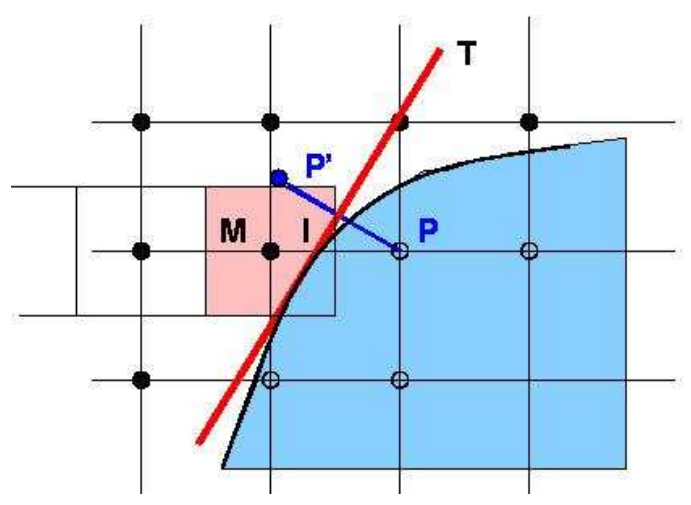

Fig. 1 Sketch of the treatment of cut cells

Since the mid-1980's, researchers have been very successful in applying Cartesian grid techniques to extremely complex geometries, including moving bodies. A good review on Cartesian grid methods was given by Aftosmis [2] and examples of application to moving boundaries can be found in [13]. Our code is based on the AMR [3] method using fixed structured mesh blocks. An essential point is the treatment of the boundary conditions on solid walls cutting the grid. As we have to consider bodies in relative motion with cylindrical contact surfaces we cannot rely on a description of the body surface by triangulated surface facets. Then a cut cell approach like the method developed by Yang, Causon and Ingram [18, 19], becomes very tedious for a moving body for which the intersections of the grid with the body surface must be recomputed at each time step. Instead we follow an approach developed by Forrer [8] which involves only the determination of the intersection of the lines passing through the cell centers with the body together with the local normal to the body surface.

A sketch of the method is given on Fig. 1. In order to update the cut cell whose center is $\mathrm{M}$ we use for the computation of the x-flux at the right cell boundary a fictitious cell whose center is $\mathrm{P}$ inside the solid body. The value of variables in this cell are determined by using a local symmetry principle with respect to the tangent plane $\mathrm{T}$ at the intersection $\mathrm{I}$. The values of the variables in the symmetrical cell whose center is at $\mathrm{P}$ ' are determined by trilinear interpolation between the 8 cells surrounding P'. If the trilinear interpolation is not feasible we use an interpolation between the surrounding valid nodes weighted by the inverse of the distance to P'. This approach should not be confused with a ghost cell method because the fictitious node P can be used with other values of the variables for evaluation of the $y$-flux or the z-flux in other boundary cells. Therefore, for computer implementation, these fictitious values cannot be stored in the standard location corresponding to the grid node P. As shown by Forrer [8] the method is of second order except at a few critical points where we must use the weighted interpolation technique. For a moving body all the computations are performed in a local reference frame moving with the body at the velocity of the intersection point I. 


\subsection{Solid Geometry Description}

The description of the surface of all solid bodies is based on a small number (12) of surface elements well suited to an exact representation of cylindrical contact surfaces:

- Truncated cone

- Truncated cone limited by two meridian planes

- Truncated cone limited by the intersection with two planes

- Plane quadrilateral with one side replaced by the intersection with truncated cone

- Triangle with two sides replaced by the intersection with two truncated cone having the same base

- Plane polygon

- Plane polygon with a circular hole

- Plane quadrilateral with one side replaced by a circular arc

- Disc

- Angular portion of a disc

- Crown

- Angular portion of a crown

For all these elements, the intersections with the grid lines and the normals at the intersection points are easily computed, leading at most to the solution of a second degree equation. Intersection computations are speeded up by defining bounding boxes for each element. The main difficulty is to avoid missing an intersection or counting twice an intersection close to the boundary between two adjacent elements. As the number of intersections of a solid body with a half straight line emanating from an external point should be even we can detect such anomalous situations due to round-off errors. The correction is performed thanks to the computation of a quality factor with each intersection (we use the minimum distance between the computed intersection and the edge of the element). In case of an odd number of intersections along a grid line the situation can be corrected by using the quality factors and the orientation of the normals at the intersection points in order to decide which point must be suppressed from the list of intersections.

After having computed the intersection of a grid line with all the solid bodies, the intersection points are sorted by increasing distance along the grid line and used to define a small number of patches of contiguous valid nodes the other nodes being blanked. When the projectile moves with respect to the fixed grid, some nodes which were used at time $\mathrm{n}$ become blanked at time $\mathrm{n}+1$ and they are just discarded but some nodes which were blanked at time $n$ become valid nodes at time $n+1$ and the should be reinitialized.

The reinitialization algorithm is based on:

- Relations along the characteristics between time $\mathrm{n}$ and $\mathrm{n}+1$

- Kinematic boundary conditions because the reinitialized nodes are usually adjacent to a boundary

- Interpolation between valid nodes at time $\mathrm{n}$ or $\mathrm{n}+1$

\section{Time Integration and Coupling}

Grid refinement is performed by halving the grid size between grid level 1 and $1+1$. So far we have used only an a priori defined configuration with 3 or 4 levels and only one grid per level. Above the coarse level 1 the grids are shifted periodically in order to always encompass the moving bodies. Figure 2 shows an example of a configuration with 3 grids.

The time integration for the external computation is performed by the classical recursive algorithm sketch on Fig. 3, a time step $\Delta \tau$ at the grid level 1 is followed by two times steps of size $\Delta \tau / 2$ at grid level $1+1$. One should notice that the integration at level 1 is performed for all the valid cells even if they are covered by a refined grid at level $1+1$. After completion of the time step these values are replaced by values obtained by merging the corresponding cells at level $1+1$. For a 3D computation, the cost of this useless work at level 1 is only $1 / 16$ of the work performed at level $1+1$. 
The internal ballistics computation is synchronized with the external computation at the highest external grid level. The time step is determined as the minimum of the allowable time steps for the internal and external computations according to the prescribed CFL value which is typically 0.3 to 0.4 .

The coupling between the internal computation and the external one is made easier by defining the external grid and the internal mesh so that they have a common cell interface at the highest grid level: we have only to average the flux values of the external computation in order to define a $1 \mathrm{D}$ flux condition for the internal computation.
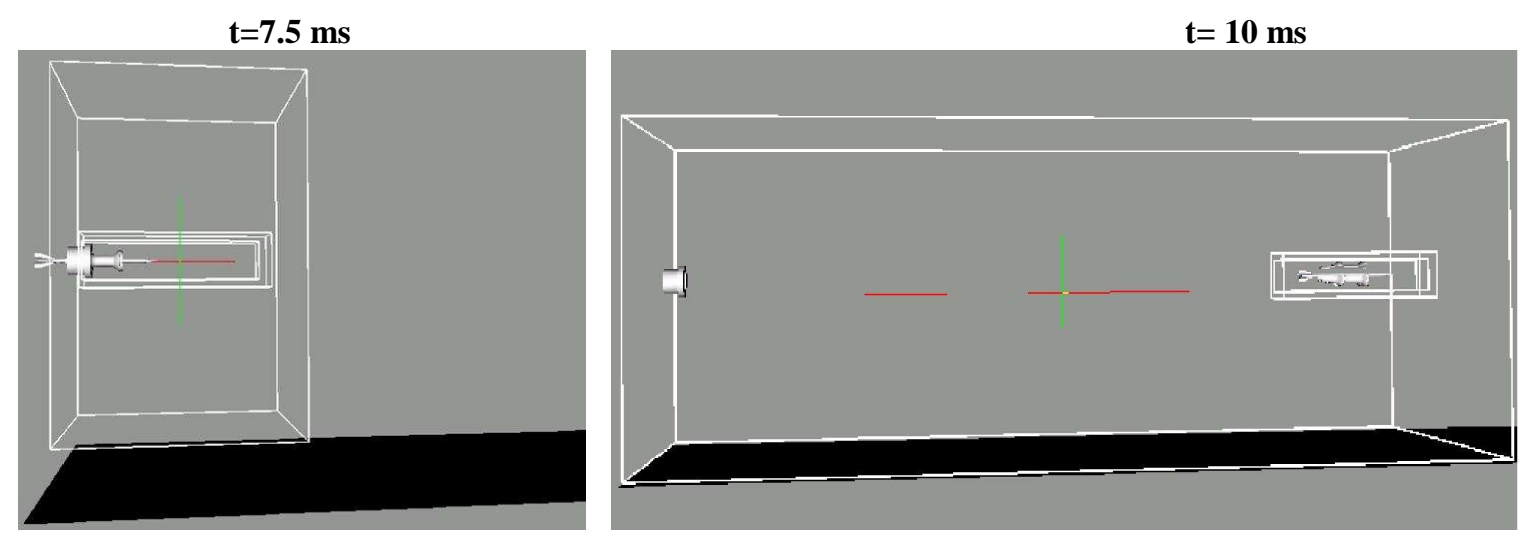

Fig. 2 Example of grid embedding with three grids: grids 2 and 3 are shifted periodically to encompass the projectile

The force and moment acting on the solid bodies are computed by integrating the pressure available at the intersection points. In fact we used only the intersection of the $\mathrm{x}$ coordinate lines for the $\mathrm{x}$ component of the force and similarly for the $\mathrm{y}$ and $\mathrm{z}$ direction. This is a feature which should be improved because only a part of the wall pressure information available is used in each direction. The forces are computed at the beginning of each time step on the highest level grid. Then the displacements of all the solid bodies are computed and the transformation matrices between the fixed reference frame and local frames linked to the bodies are updated. For the computation of the displacement of the solid rigid bodies we use a simple predictor corrector method and a Hamilton's quaternion formulation introduced in reference [5]. At the beginning of the sabot discard phase, we prescribed as initial conditions the radial velocity and pitching rate of the three sabot petals given by the dynamical elasticity code LS-DYNA.

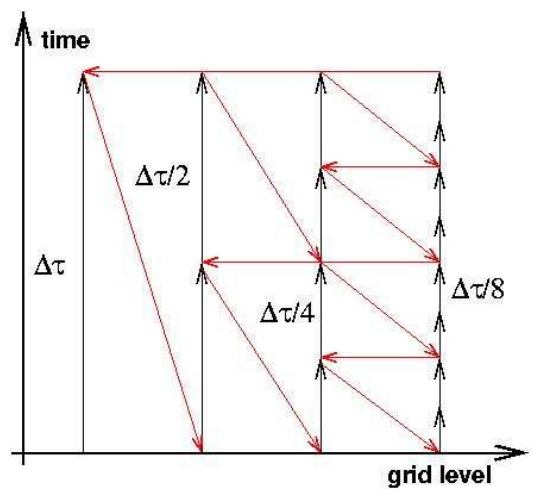

Fig. 3 Sketch of the recursive time integration scheme for a four level mesh 


\section{Validation Results}

\subsection{Validation with Academic Test Problems}

Validation of this rather complex code is of course essential. Unfortunately there are very few threedimensional exact solutions of the Euler equations and we have chosen to use classical one-dimensional (1D) shock tube problems in a 3D context: as shown on Fig. 4 we put in the external field, after the gun muzzle, a closed tube of circular cross section which can be oriented at an angle $\alpha$ with respect to the $\mathrm{x}$ axis.

The geometrical parameters are the ratio D/L between the diameter and the length of the tube and the angle $\alpha$. Inside the tube, the field is initialized with the conditions corresponding to well known 1D test problems [11] and the challenge is to recover the $1 \mathrm{D}$ solution when tube axis is not aligned with the $\mathrm{x}, \mathrm{y}$ or $\mathrm{z}$ directions. We have use all the 1D test cases defined in reference [11]. Figure 5 shows the results for Toro's test case 1, a shock tube problem with a sonic point in the rarefaction. On this graph we have plotted the values of all the nodes inside the tube. Figures 6 and 7 allow to compare the results with $\alpha=0$ and $\alpha=45$ for the classical WoodwardCollela blast wave problem. For this last case there is no analytical solution and the solution labeled exact is obtained with a fifth order highly accurate scheme with 2000 nodes.

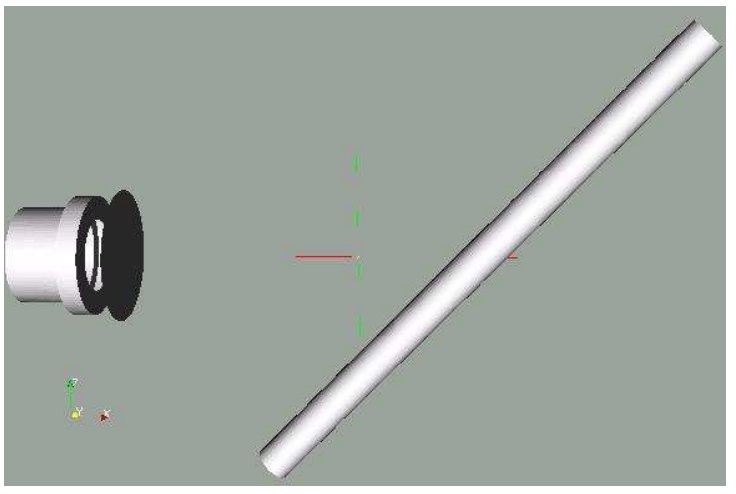

Fig. 4 Configuration for 1D tests in a 3D context

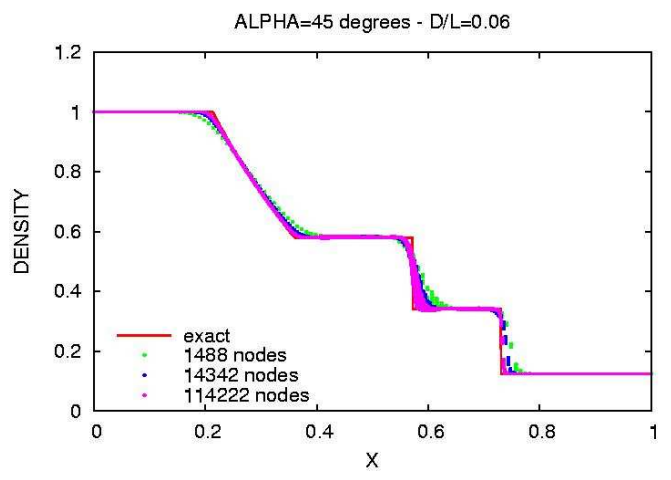

Fig. 5 TORO's test 1 in a tube with $D / L=0.6$ and $\alpha=45$ degrees computed wit $C F L=0.5$ 


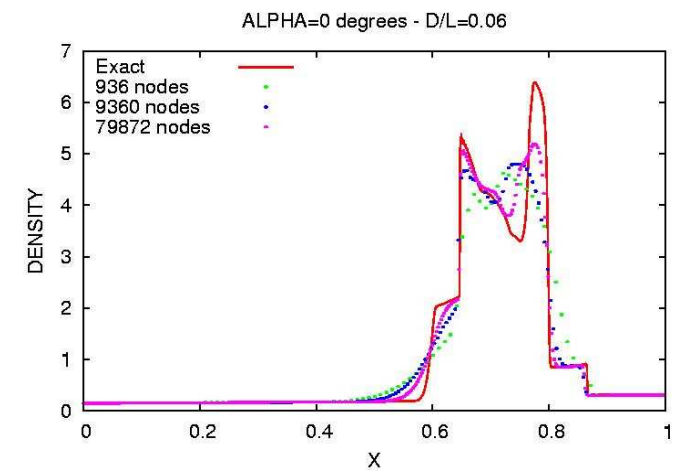

Fig. 6 Woodward-Colella blast wave problem in a tube with $D / L=0.06$ and $\alpha=0$ degrees

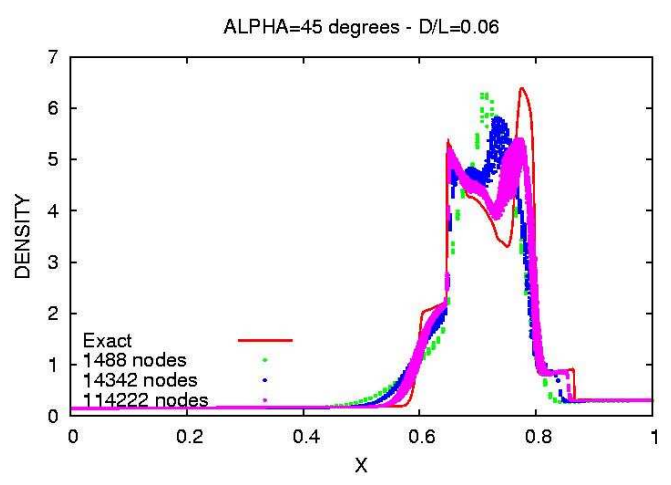

Fig. 7 Woodward-Colella blast wave problem in a tube with $\mathrm{D} / \mathrm{L}=\mathbf{0 . 0 6}$ and $\alpha=45$ degrees

\subsection{Experimental Firing Set-Up and Test Conditions}

Experimental investigation of the phenomena occurring during gun propulsion, sabot discard and blast wave propagation is costly and difficult in view of the short time, high pressure and temperature ranges and very severe environmental conditions. The tests were conducted in the French MOD ETBS Test Center of Bourges. Firing experiments were performed with an advanced $120 \mathrm{~mm}$ lightweight tank demonstrator with a smoothbore tube, a muzzle brake, and launching APFSDS projectiles (see Fig. 8). For validation purposes, detailed experiments were conducted in 2009. The interior ballistics was investigated by means of pressure chamber and muzzle velocity measurements. We investigated the intermediate ballistics, muzzle brake flow, sabot discard, and muzzle blast interaction with the tank demonstrator using high-speed cameras, and pressure measurements. These were taken on the ground, at a certain height, and on the demonstrator.

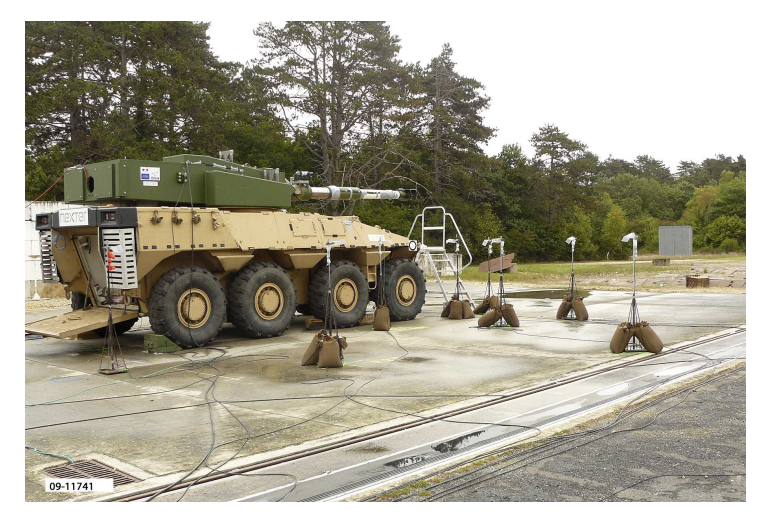

Fig. $8120 \mathrm{~mm}$ lightweight tank demonstrator 


\subsection{Grids and Calculation Costs}

One important problem is that the computational domain is very large. Different grids were built, for example in the case of the computation of the muzzle brake flow and of the intermediate ballistics flow, the external dimensions of the 3D computational fluid domain are $11 \mathrm{~m} \times 6 \mathrm{~m} \times 4 \mathrm{~m}$. In this case, the Cartesian grid is constituted of $18 \times 10^{6}$ nodes, local grid refinement is performed by halving the grid size between grid level 1 and $1+1$. As presented in Fig. 9, we have used 6 grid levels with a minimum spatial step size $\Delta x=\Delta y=\Delta z$ of about $2 \mathrm{~mm}$. In spite of the high number of nodes the real geometry was necessarily simplified (in particular the description of the tank vehicle) in order to achieve the computation.

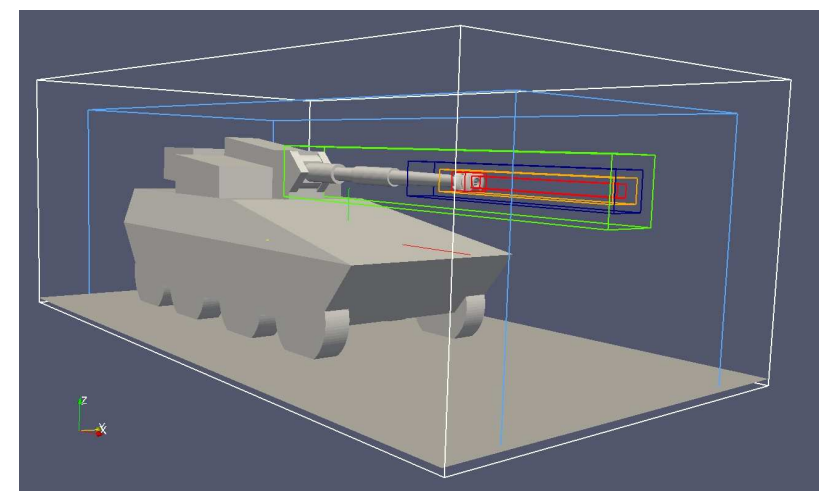

Fig. 9 Lightweight tank demonstrator, example of grid with 6 levels

Unsteady computations are performed with a 4 bi-proc Xeon $(3.06 \mathrm{GHz})$ cluster under Windows ${ }^{\circledR} 2000$. The average calculation cost is about $0.5 \mu$ s/node/iteration.

\subsection{Internal Ballistics Validation}

Figure 10 gives an example of the pressure distributions in the tube for a $120 \mathrm{~mm}$ demonstrator smooth-bore barrel launching an APFSDS projectile. On the left, the pressure evolution, every $0.2 \mathrm{~ms}$, between the breech (at the abscissa $X=0 \mathrm{~m}$ ) and the projectile is presented. On the right, the pressure evolution, also every $0.2 \mathrm{~ms}$, between the projectile and the gun muzzle (at the abscissa $X=6.25 \mathrm{~m}$ ) is illustrated.
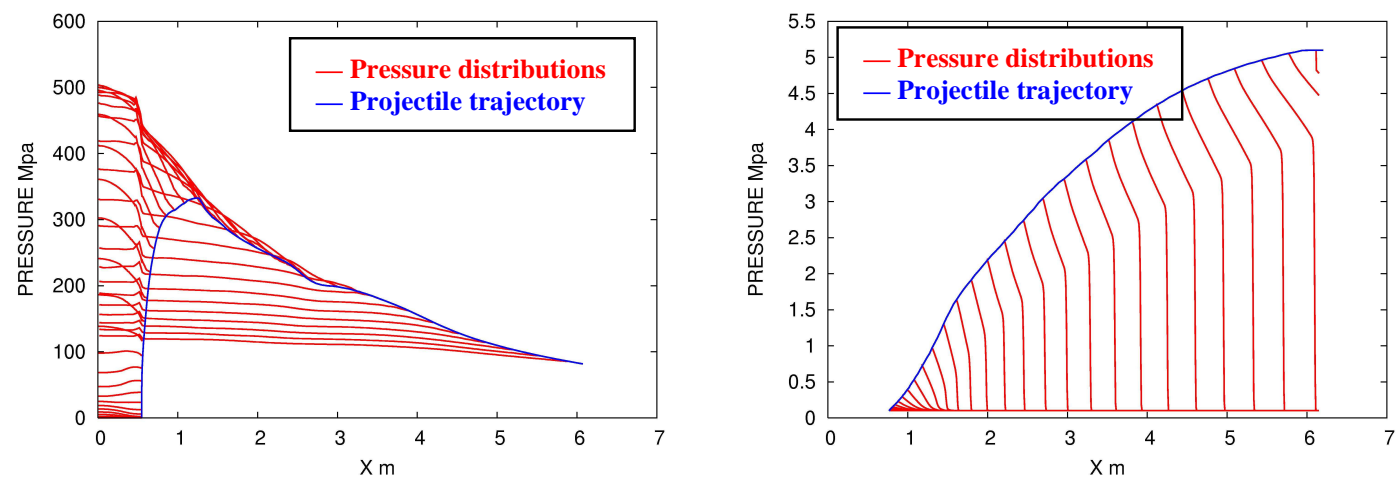

Fig. 10 Pressure distributions, every $0.2 \mathrm{~ms}$, between the breech and the projectile, and between the projectile and the gun muzzle 


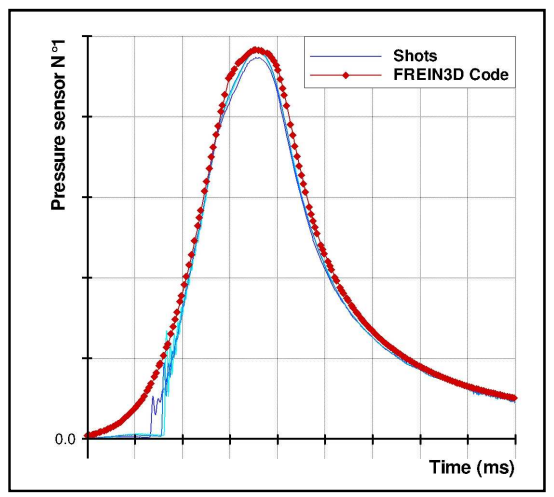

Fig. 11 Experimental and theoretical pressure evolutions with time in the chamber at $\mathbf{2 . 4 6}$ calibers from the breech

The evolution of the internal pressure over time in the gun chamber is presented in Fig. 11. The level and shape of the pressure signal are fairly well reproduced. The relative errors on the maximum pressure are less than $6 \%$, and the initial velocity is predicted to $0.16 \%$. Note that, the usual error on the prediction of initial velocity is a few percent. The agreement on the prediction of the ignition phase is less satisfactory this is due to the fact that, in the first moments, the combustible cartridge blocks the pressure sensor.

\subsection{Muzzle Brake Flow Validation}

Figure 12. is an example of computation of the firing of an APFSDS through the muzzle brake of the $120 \mathrm{~mm}$ lightweight tank demonstrator.

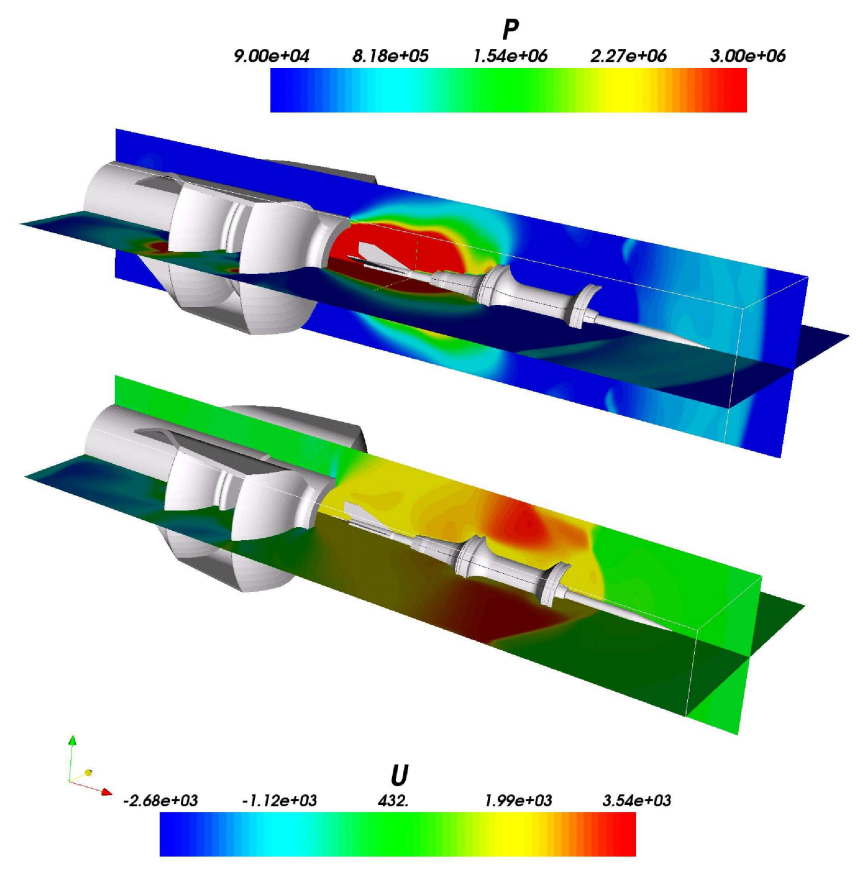

Fig. 12 Firing of a $120 \mathrm{~mm}$ APFSDS through a muzzle brake, pressure field and $u$ component of the velocity

The projectile is still very close to the muzzle brake and surrounded by the propellant gas which expands at a much higher speed than the projectile. Expansion around the sabot generates low pressures in front of the sabot and projectile, and for a short distance, the projectile is still accelerated by the gas. A significant loss of 
symmetry between the vertical plane $\mathrm{y}=0$ and the horizontal plane $\mathrm{z}=0$ is observed due to the flow of the propellant gas through the lateral blowholes which after deflection by the muzzle brake blades generates a negative $\mathrm{x}$ component of the velocity at the exit from the brake.

Figure 13 compares the computed pressure with the data from a pressure sensor located at about $1.6 \mathrm{~m}$ from the tube axis in the muzzle exit plane. The level and shape of the pressure signal are very well reproduced. Complementary validation results are presented in details in References [29, 31, 32].

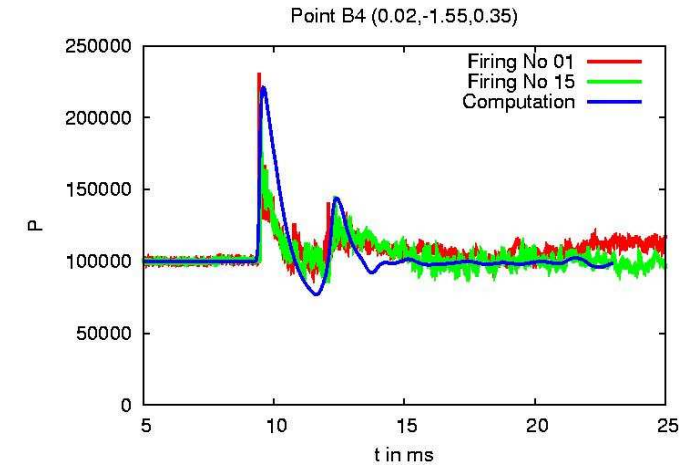

Fig. 13 Pressure in the muzzle exit plane at $1.6 \mathrm{~m}$ from the tube axis

\subsection{Sabot Discard Validation \\ 4.6.1 Quasi 2D Approximation and Tests for a $44 \mathrm{~mm}$ Gun}

In order to investigate, at a reasonable computational cost, the grid convergence problem we have developed a $2 \mathrm{D}$ axisymmetric version in cylindrical coordinates.

In this case the penetrator shape should be axisymmetric (no fins) and the sabot is represented as a dilatable obstacle which expands radially while keeping the shape of its cross section in a meridian plane. The flow between the petals and the projectile is taken into account but the flow between the petals is not represented. In order to compute the forces acting on the petals, the pressure on the plane sides must be empirically interpolated. This approximation is valid only at the beginning of the sabot discard and when the petals reach a high incidence angle the computation strongly overestimates the pressure in front of the sabot system and the drag.

This quasi axisymmetric approximation was used to compare three sabot shapes for a $44 \mathrm{~mm}$ gun for which Xray visualizations of the sabot discard were available. In the first configuration, shown on Fig. 14, the front part of the sabot incorporates a cavity which generates high pressures and a positive pitching moment. The petals quickly reach high positive incidences which are reproduced in the computation. For the second configuration, shown on Fig. 15, the sabot incorporates two cavities, one at the front and one at the base. Initially, the high pressures generated by the propellant gas in the base region generate a negative pitching moment which is later replaced by a positive pitching moment due to the front cavity: the petals separate from the penetrator at a small negative incidence angle which thereafter becomes positive thereby minimizing the risk of contact between the petals and the penetrator. Here again the computation agrees fairly well with the experiment. In the last configuration, shown on Fig. 16, the front part is flat and only the base cavity is used: in that case, the petals reach large negative incidence angles with contact in the front part between the petal and the penetrator. In this last case the agreement with the computation is less satisfactory. 


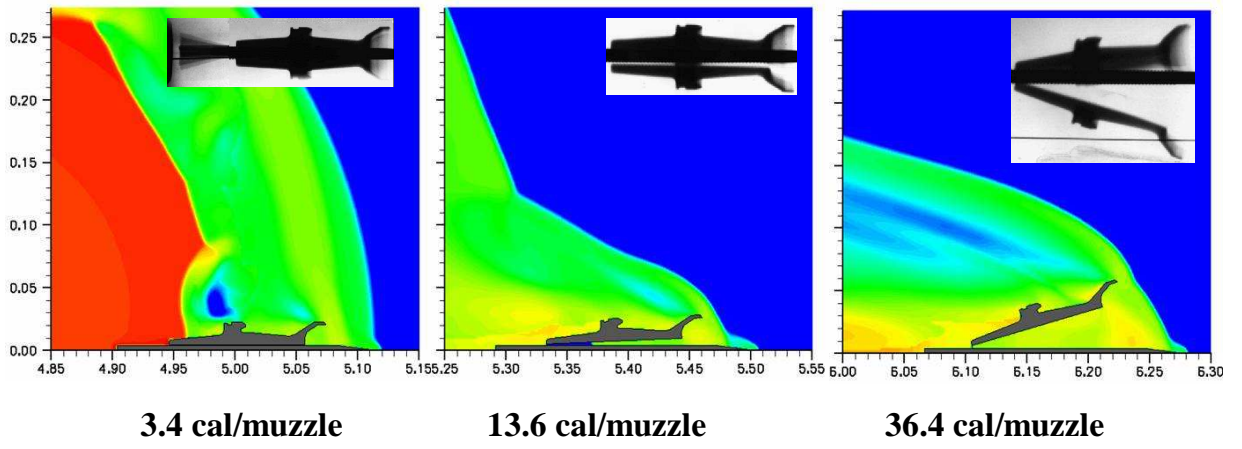

Fig. 1444 mm gun - sabot discard for sabot type 1

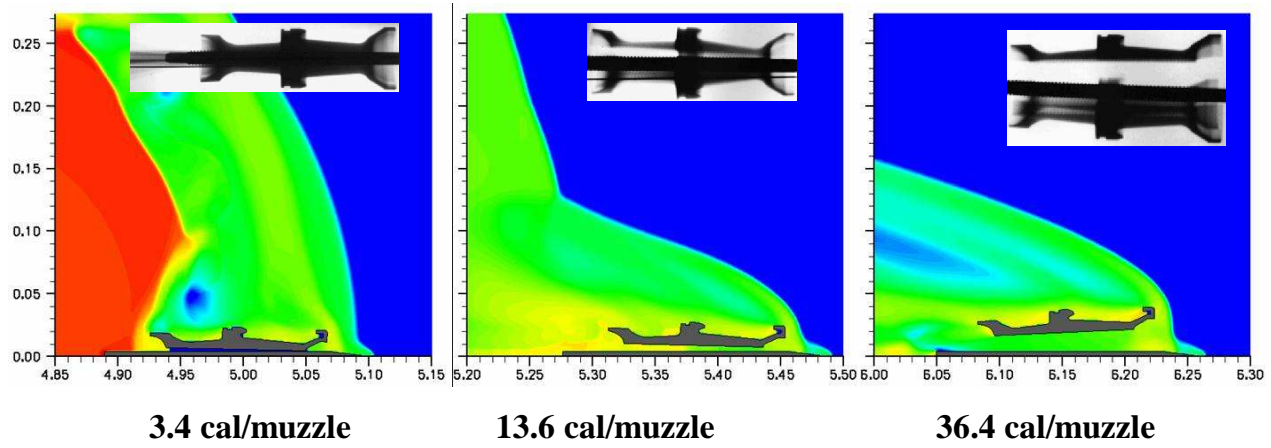

Fig. 1544 mm gun - sabot discard for sabot type 2

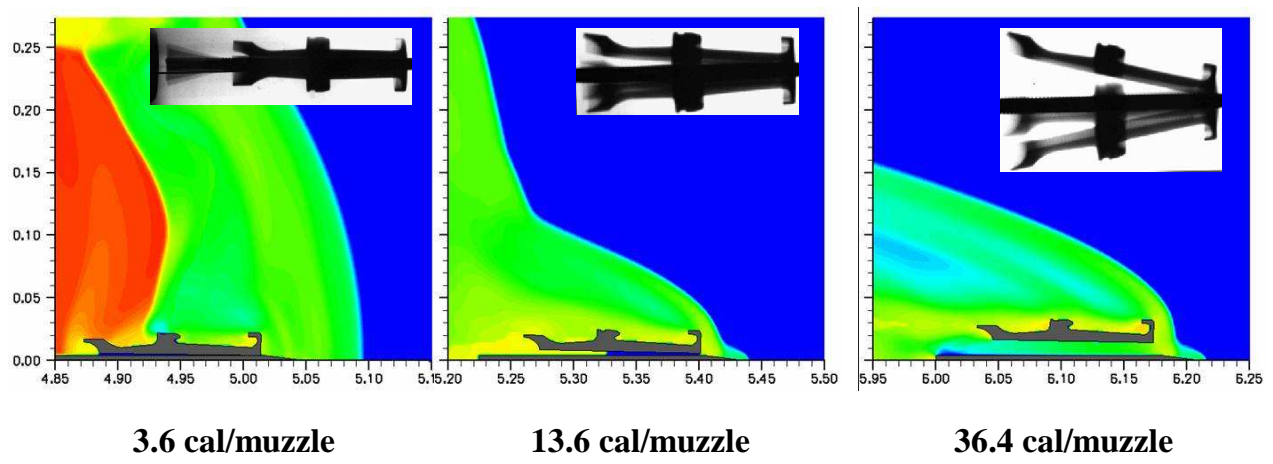

Fig. 1644 mm gun - sabot discard for sabot type 3

\subsubsection{D Sabot Discard Validation}

Optimization of the sabot shape in order to avoid solid contact with the projectile during the separation and to minimize the aerodynamics perturbations is a rather difficult task in view of the numerous constraints and the complexity of the phenomena involved. For classical projectiles, a common practice way is to use a muzzle brake in order to reduce the mass of the gun recoil system. It has always been of interest to fire APFSDS projectiles through a muzzle brake. However, this is a device which involves several blow-holes with blades which deflect the propellant gas backward therefore reducing the total impulse communicated to the gun. In the present state of knowledge of the intermediate ballistics phenomena, firing an APFSDS through a muzzle brake adds a high risk of inducing large perturbations on the projectile when the fins pass in front of the blow-holes. 
The computation was performed with 6 embedding grids using $12 \times 10^{6}$ nodes. The external dimensions of the $3 \mathrm{D}$ computational fluid domain are $23 \mathrm{~m} \times 3 \mathrm{~m} \times 3 \mathrm{~m}$. However only the sabot petals movement was computed and the penetrator was forced to move along the tube axis at a zero incidence angle. The reason is that strict respect of isotropy was not achieved on the fins: as the fins are beveled they generate aerodynamic forces which, in an otherwise quasi symmetrical situation, should generate only a roll moment without lift or lateral force. The configuration involves fins of which, during the sabot discard, three are located in the wake of the sabot petals, therefore generating negligible forces.

The three other fins are subjected to the high speed flow between the three petals and generate much larger aerodynamic contributions. One of these three fins is aligned with the grid while the other two make an angle of 30 degrees with the y axis. As they are not seen in the same way on the Cartesian mesh, they generate slightly different forces from the first one. Summing the contribution of all the fins gives a resulting lateral force which may perturb the projectile in flight. This is clearly a point which should be understood because the lever arm between the fins and the center of mass of the penetrator is large and strict maintenance of the symmetry is essential in order to be able to compute small perturbations with respect to the ideal configuration. The computed intermediate wave systems (shock and expansion in grey), pressure applied on the muzzle brake, on the sabot components and on the projectile (in color), and the experimental and numerical unsteady 3D discard are presented in Fig. 17.
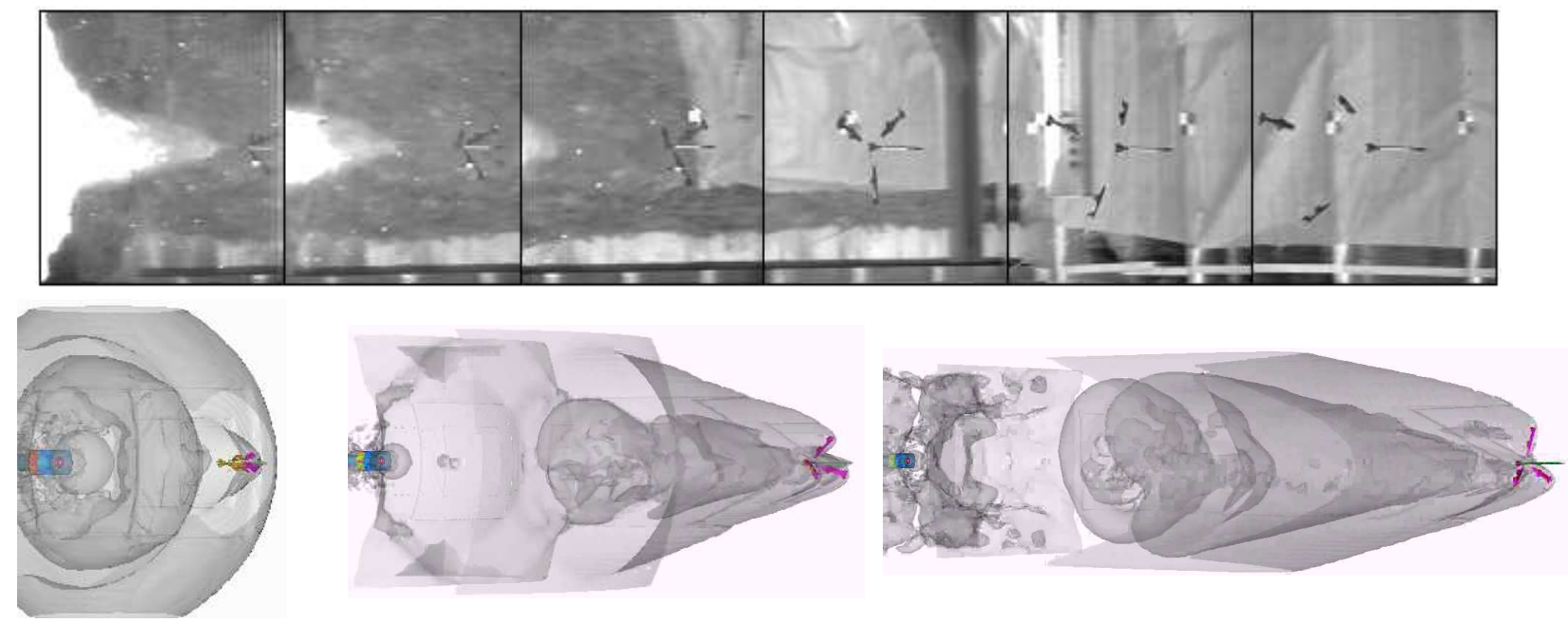

Fig. 17 Experimental and numerical 3D unsteady sabot discard

Figure 18 shows the comparison between the computation and the experiments for the sabot pitch angle and the distance between the sabot and the penetrator. Up to a pitch angle of 50 degrees the agreement is fairly good and the discrepancy for larger angles is due to the modeling approximations which overestimate the pressure in front of the sabot petals and the pitching moment. Validation results are presented in References [28, 29, 31, 32]. 

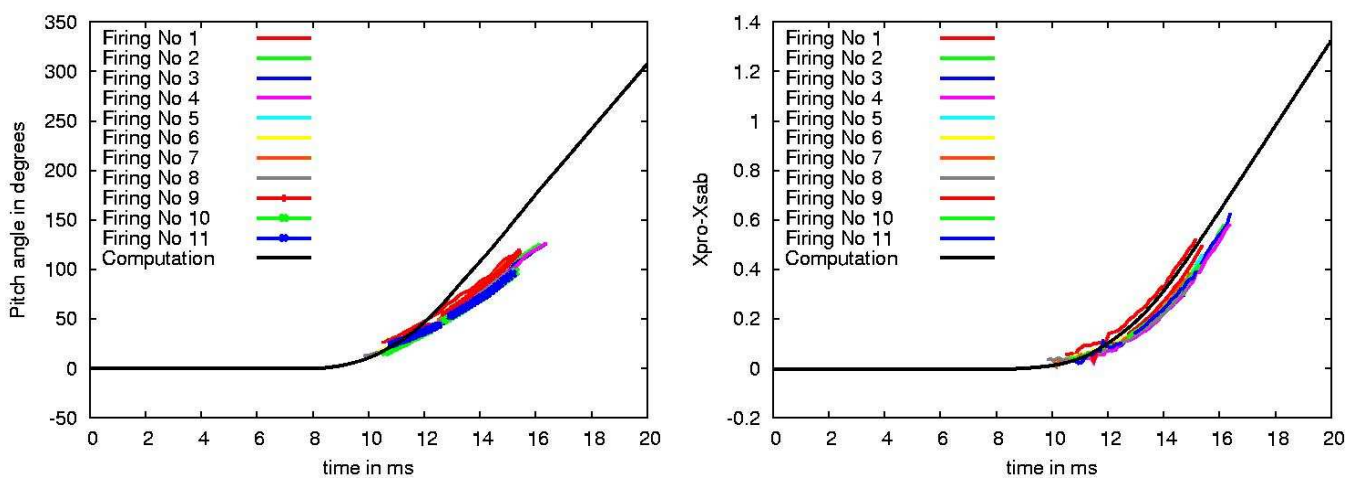

Fig. 18 Time evolution of the sabot angle and of the $\mathrm{x}$ separation between penetrator and sabot

4.7 Prediction of the Unsteady Pressure Field Around the Tank Demonstrator

Locations of the experimental pressure sensors on the tank vehicle are presented on Fig. 19.
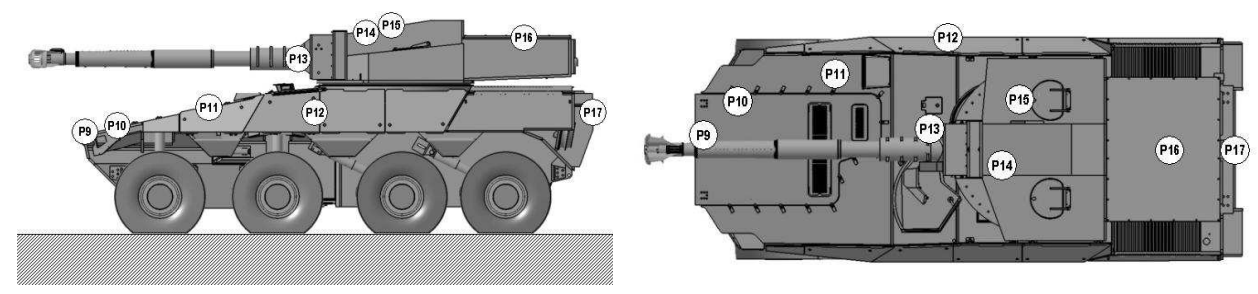

Fig. 19 Pressure measurement locations on the tank demonstrator

Example of the computed unsteady pressure environment computation is presented on Fig. 20, the intermediate wave systems and the pressure applied on the tank demonstrator are represented, as are the blast wave reflection on the ground and on the tank demonstrator.
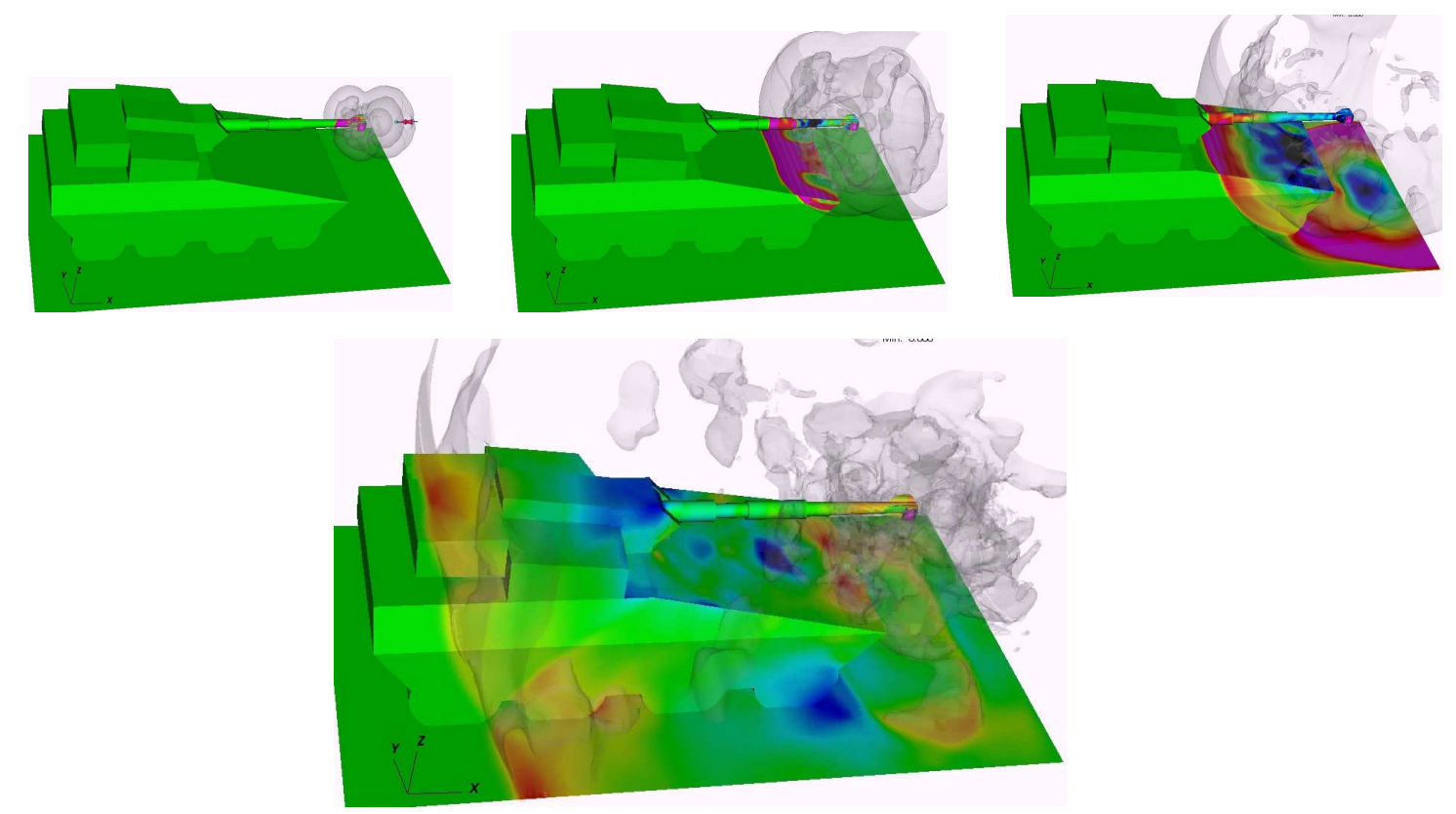

Fig. 20 Pressure contours on the tank demonstrator at different time levels 
As example, Fig. 21 compares the computed pressure with measurements from pressure sensors located on the front part of the tank vehicle at points $\mathrm{N}^{\circ} 9,10,12$ and 17.
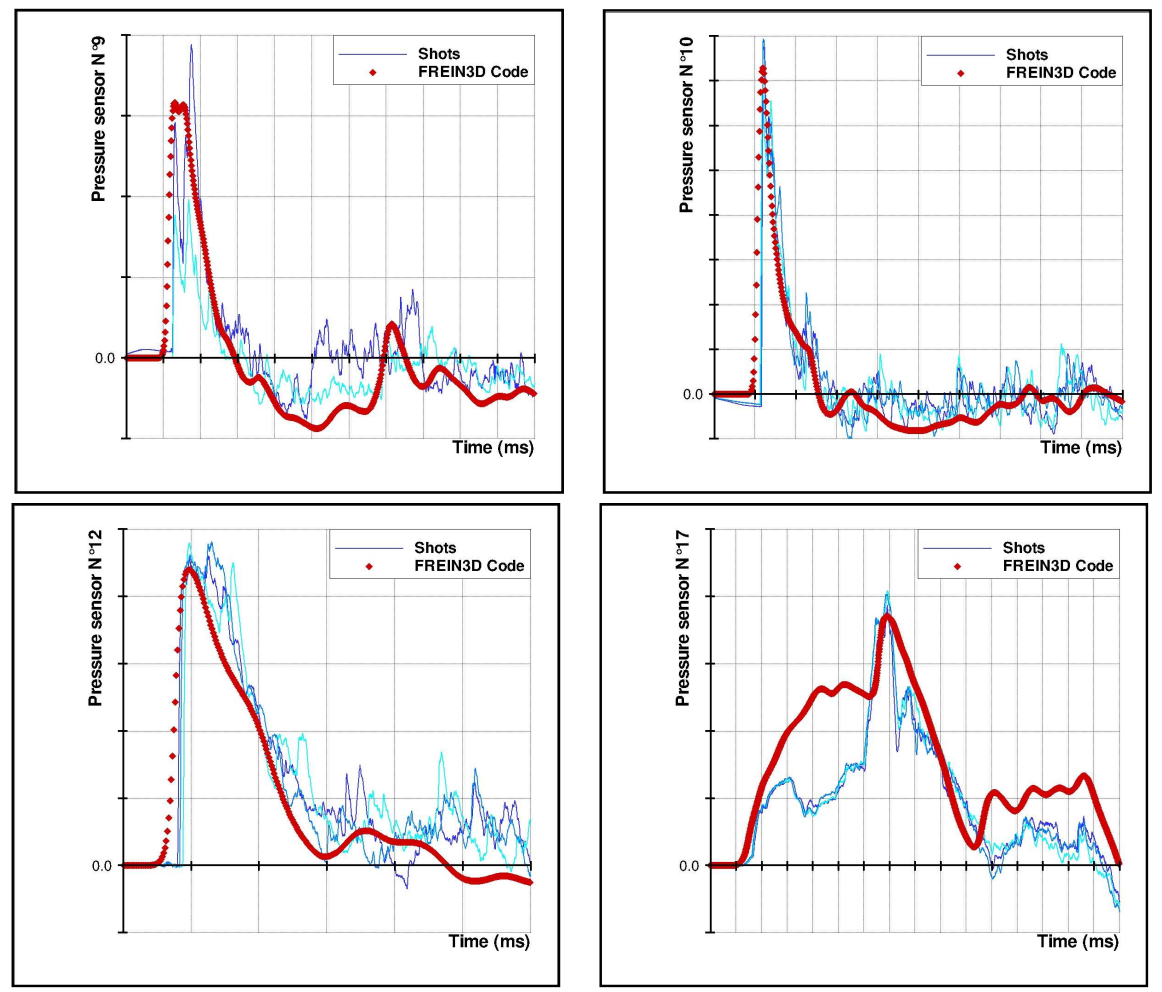

Fig. 21 Pressure versus time for measurement points $N^{\circ}$ 9, 10, 12 and 17

Note that the pressure time evolutions are synchronized to the time of the first maximum overpressure. The measured values were generally slightly higher than the numerical simulation. A satisfactory qualitative and quantitative agreement was obtained.

The average relative error on the prediction of the reflected overpressure is less than $5.5 \%$ and the relative errors are always less than $8 \%$ percent. Even at the back of the tank demonstrator, for the pressure sensor location 17 the agreement is acceptable.

Despite the low number of test repetitions, the differences observed between the firing results of the test campaign, the global simplification of the modeling and the fact that the grid optimization is not fully reach, we can consider that the agreement is globally very good. More complete validation results are presented in Reference [32].

\subsection{Prediction of the Unsteady Exterior Ballistics}

During the initial phase of the sabot discard, the projectile is subjected to the rapid expansion of the propellant gas and the velocity of the fluid with respect to the projectile is negative. Then the projectile overtakes the blast wave system and begins its flight at a high supersonic Mach number. Strong unsteady interference between the sabot petals, the projectile and the blast wave system generate aerodynamic forces which can perturb the initial conditions of flight and induce on-target dispersion. 
Another source of dispersion comes from the gun tube dynamic motion and from the possible transverse motion of the projectile in the tube called balloting. These sources of perturbation could be evaluated by structural dynamic computations and introduced as muzzle initial conditions in the fluid dynamics model.

The theoretical and numerical approaches that have been developed could give the unsteady aerodynamics of the projectile in flight. But at this time only the Euler equations are solved and a natural improvement would be to substitute Euler by Navier-Stokes equations. Figure 22 shows the pressure and the temperature around the penetrator flying alone, after the sabot discard, at about $1760 \mathrm{~m} / \mathrm{s}$.

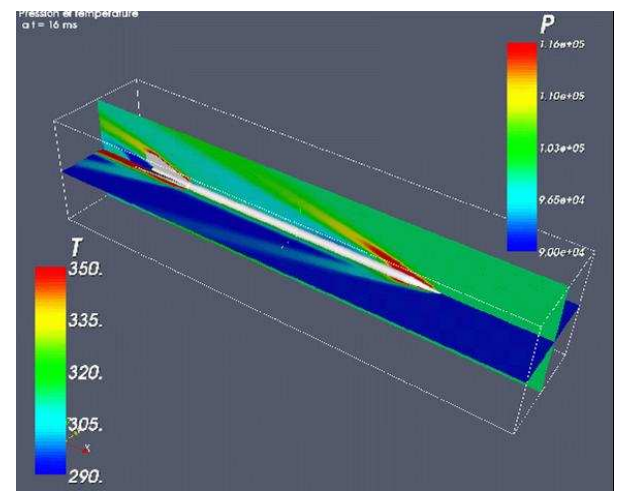

Fig. 22 Penetrator in flight after sabot discard at $\mathrm{t}=16 \mathrm{~ms}$

\section{Conclusion}

The present results show that the computation of such real 3D complex situation involving numerous physical phenomena, and a coupling between fluid dynamics and flight mechanics (and also structural dynamics) is now feasible, at least for the Euler equations.

Globally the validation results are very satisfactory.

Nevertheless, improvement of the 3D modeling is needed before reaching the goal of predicting the overall initial perturbations of the projectile trajectory.

The main improvements needed are:

- to advance the mesh refinement technique because the flow involves very complex features like RichtmyerMeshkov type instabilities which could have an influence on the projectile trajectory. Attempt to refine the mesh using a local error estimate leads to a prohibitive number of nodes which are not useful if the purpose is only the prediction of the forces and trajectories. In that case we clearly need a mesh refinement algorithm which takes into account the objectives of the calculation. Also, the loss of isotropy on a Cartesian grid in the presence of a body having a 120 degree symmetry is a difficult problem which remains essential for the prediction of the moment acting on fin-stabilized projectiles.

- to substitute Euler equations by Navier-Stokes equations.

- to develop a link between the FREIN code and industrial CAD geometry software in order to increase the operational capacity of the code.

- developments are also needed, for example, to take account of the gun recoil effect, etc.

Complementary experimental studies are also necessary in order to widely validate the theoretical and the numerical approaches.

\section{Acknowledgments}

The authors would like to thank Eric Cavallari, Philippe Bidorini and Serge Secco, from the French MOD UMTER Program Division for their support in this work. The authors also thank the ETBS Test Center Team for their strong cooperation in this study. 


\section{Reference}

\section{Theoretical References}

[1] Abgrall, R., and Karni, S., 2001, “Computation of Compressible Multifluids,” Jour. Comp. Phys., 169, pp. 594-623.

[2] Aftosmis, M. J., 1997, "Solution Adaptative Cartesian Grid Methods for Aerodynamic Flows with Complex Geometries," Proc. 28 ${ }^{\text {th }}$ Computational Fluid Dynamics Lecture Series, Von Karman Institute for Fluid Dynamics, 1997-02.

[3] Berger, M. J., and Collela, P., 1989, "Local Adaptative Mesh Refinement for Shock Hydrodynamics," Jour. Comp. Phys., 82, pp. 64-84.

[4] Champigny, P., d'Espiney, P., and Ceroni, D., 1998, "Computation of Sabot Discard Using Chimera Technique," Proc. 17th International Symposium on Ballistics, Midrand, South Africa, Vol. 2, pp. 113-120.

[5] Dietrich, F., Guillen, Ph., and Cayzac, R., 2003, "A Flight Mechanics/ Aerodynamics Coupling Methodology for Projectiles," Proc. $41^{\text {st }}$ AIAA Aerospace Sciences Meeting \& Exhibit, Reno, USA, Paper 0029.

[6] Drew, D. A., 1983, "Mathematical Modeling of Two-Phase Flow,” Ann. Rev. Fluid. Mech., 15, pp. 261291.

[7] Ferry, E. N., Sahu, J., and Heavy, K. R., 1996, "Navier-Stokes Computations of Sabot Discard Using Chimera Scheme," Proc. $16^{\text {th }}$ International Ballistics Symposium and Exhibition, San Francisco, USA, Vol. 2, pp. 13-22.

[8] Forrer, H., 1996, "Second Order Accurate Boundary Treatment for Cartesian Grid Methods," Seminar f"ur Angewandte Mathematic, ETH Z"urich, ETH Research Report No. 96-13, see http://www.sam.math.ethz.ch/Reports/1996-13.html.

[9] Gough, P. S., and Zwarts, F. J., 1979, "Modeling Heterogeneous Two-phase Reacting Flow," AIAA Jour., 17(1), pp. 17-25.

[10] Kim, S., Kim, C., Rho, O., and Kyu Hong, S., 2003, "Cure for the Shock Instability : Development of a Shock-Stable Roe Scheme,” Jour. Comp. Phys., 185, pp. 342-374.

[11] Liska, R., and Wendroff, B., 2003, "Comparison of Several Difference Schemes on 1D and 2D Test Problems for the Euler Equations,” SIAM J. Sci. Comp., 25(3), pp. 995-1017.

[12] Mulder, W., Osher, S., and Sethian, J., 1992, "Computing Interface Motion in Compressible Gas Dynamics," Jour. Comp. Phys., 100, pp. 209-228.

[13] Murman, S. M., Aftosmis, M.J., and Berger, M. J., 2003, "Implicit Approaches for Moving Boundaries in a 3-D Cartesian Method," 41st AIAA Aerospace Sciences Meeting, Reno, USA, AIAA-2003-1119.

[14] Pokharna, H., Mori, M., and Ransom, V., 1997, "Regularization of Two-Phase Flow Models: a Comparison of Numerical and Differential Approaches," Jour. Comp. Phys., 134, pp. 282-295.

[15] Roe, P.L., 1981, "Approximate Riemann Solvers, Parameter Vectors and Difference", Schemes Jour. Comp. Phys, 43, pp. 357-372.

[16] Sainsaulieu, L., 1995, "Finite Volume Approximation of Two-Phase Fluid Flows Based on an Approximate Roe-Type Riemann Solver,” Jour. Comp. Phys., 121, pp. 1-28.

[17] Stewart, H. B., and Wendroff, B., 1984, "Two-Phase Flow : Models and Methods," Jour. Comp. Phys, 56, pp. 363-409.

[18] Yang, G., Causon, D. M., Ingram, D. M., Saunders, R., and Batten, P., 1997, "A Cartesian Cut Cell Method for Compressible Flows. Part A : Static Body Problem," Aeronautical Journal, 101(1002), pp. 57-65.

[19] Yang, G., Causon, D. M., and Ingram, D. M., 1997, "Calculation of 3D Compressible Flows Around Moving Bodies," Proc. 21st International Symp. on Shock Waves, Great Keppel Island, Australia, paper 1780.

[20] Klingenberg, K., and Heimerl, J. M, 1992, "Gun Muzzle Blast and Flash,” Progress in Astronautics and Aeronautics, Vol. 139.

[21] Alziary de Roquefort, T., Cayzac, R., and Carette, E., 2007, "Balistique Intermédiaire pour des Projectiles Flèche,” Proc. $42^{\text {éme }}$ Colloque d'Aérodynamique Appliquée, AAAF, Nice, France.

\section{Application References}

[22] Cayzac, R., Mennechet, A, and Carette, E., 1995, "Sources of Dispersion Occuring with APFSDS Launch Dynamics," Proc. 15th International Symposium on Ballistics, Jerusalem, Israel, Vol. 3, pp. 265-273. 
[23] Cayzac, R., Vaglio, C., Brossard, J., Carette, E., and Alziary de Roquefort, T., 1996, "Transitional Ballistic Investigations," Proc. 47th Aeroballistic Range Association Meeting, ISL, Saint-Louis, France.

[24] Cayzac, R., Vaglio, C., Brossard, J., Carette, E., and Alziary de Roquefort, T., 1998, "Intermediate Ballistic Computations and Comparison with Firing Tests," 17th International Symposium on Ballistics, Midrand, South Africa, Vol. 2, pp.1-8.

[25] Boisson, D., Cayzac, R., and Légeret, G., 1999, "Study of the Heat Exchanges Occuring During the Cooling Phase in a Gun Barrel - Validation," 18th International Symposium on Ballistics, San Antonio, USA.

[26] Boisson, D., Cayzac, R., and Légeret, G., 2000, "Study of the Gas Discharge and the Heat Exchanges Occuring in a Gun Barrel After the Projectile Leaves the Barrel - Validation for the $30 \mathrm{~mm}$ Gun," Proc. European Forum on Ballistics of Projectiles, Institute Saint Louis, Saint Louis, France, pp. 129-135.

[27] Cayzac, R., and Carette, E., 2000, "Intermediate Ballistics and Aeroballistics Overview," Proc. European Forum on Ballistics of Projectiles, Institute Saint Louis, Saint Louis, France, pp. 259-274.

[28] Cayzac, R., Carette, E., and Alziary de Roquefort, T., 2001, "Intermediate Ballistic Unsteady Sabot Separation: Computations and Validations," Proc. 19th International Symposium on Ballistics, Interlaken, Switzerland, Vol. 1, pp. 297-305.

[29] Cayzac, R., Carette, E., Alziary de Roquefort, T., Bidorini, P., Bret, E., Delusier, P., and Secco, S., 2005, "Unsteady Intermediate Ballistics: 2D and 3D CFD Modeling, Applications to Sabot Separation and Blast Behaviour," Proc. $22^{\text {nd }}$ International Symposium on Ballistics, Vancouver, Canada, Vol.1, pp. 398-404.

[30] Kurbatskii, A. K., Montanari, F., Cler, D. L., and Doxbeck, M., 2007, "Numerical Blast Wave Identification and Tracking Using Solution-Based Mesh Adaptation Approach," Proc. $18^{\text {th }}$ AIAA computational Fluid dynamics Conference, Miami, USA, AIAA 2007-4188.

[31] Cayzac, R., Carette, E., and Alziary de Roquefort, T., 2008, "3D Unsteady Intermediate Ballistics Modeling: Muzzle Brake and Sabot Separation," Proc. $24^{\text {th }}$ International Symposium on Ballistics, NewOrleans, USA, Vol. 1, pp. 399-405.

[32] Cayzac, R., Carette, E., Alziary de Roquefort, T., Renard, F. X., Roux, D., Patry, J. N., and Balbo, P., 2010, "Advanced $120 \mathrm{~mm}$ Lightweight Tank Demonstrator: Direct Modeling of the Interior, Intermediate and Exterior Ballistics as well as the Weapon System Environment," Proc. 25 ${ }^{\text {th }}$ International Symposium on Ballistics, Beijing, China, Vol. 1, pp. 505-511. 